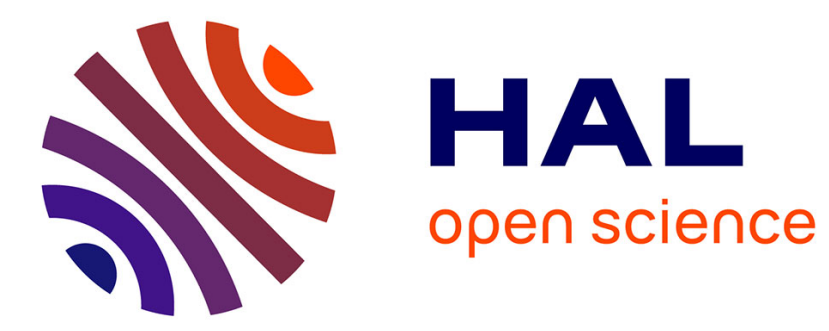

\title{
A versatile fast-development platform applied to closed-loop diaphragmatic pacing
}

Adeline Zbrzeski, Ricardo Siu, Yannick Bornat, Brian Hillen, Ranu Jung, Sylvie Renaud

\section{- To cite this version:}

Adeline Zbrzeski, Ricardo Siu, Yannick Bornat, Brian Hillen, Ranu Jung, et al.. A versatile fast-development platform applied to closed-loop diaphragmatic pacing . 7th International IEEE/EMBS Conference on Neural Engineering (NER), Apr 2015, Montpellier, France. pp.791-794, 10.1109/NER.2015.7146742 . hal-01347976

\section{HAL Id: hal-01347976 https://hal.science/hal-01347976}

Submitted on 22 Jul 2016

HAL is a multi-disciplinary open access archive for the deposit and dissemination of scientific research documents, whether they are published or not. The documents may come from teaching and research institutions in France or abroad, or from public or private research centers.
L'archive ouverte pluridisciplinaire HAL, est destinée au dépôt et à la diffusion de documents scientifiques de niveau recherche, publiés ou non, émanant des établissements d'enseignement et de recherche français ou étrangers, des laboratoires publics ou privés. 


\title{
A versatile fast-development platform applied to closed-loop diaphragmatic pacing
}

\author{
A.M. Zbrzeski, R. Siu, Y. Bornat, B.K. Hillen, R. Jung, Senior Member, IEEE, S. Renaud, Member, \\ IEEE
}

\begin{abstract}
People with cervical spinal cord injury have partial or complete loss of ventilatory control and require ventilator assist. Open-loop diaphragmatic pacing can be utilized to provide this assist. A closed-loop diaphragmatic pacing system could overcome the drawbacks for manual titration of the stimulation and respond to changing ventilatory requirements. We have developed a versatile custom hardware platform dubbed "Multimed" for biosignal acquisition and parallel real-time computation, data display and storage. We have also developed a new rodent model for diaphragmatic pacing. Using these we illustrate, to our knowledge for the firsttime, the successful ability to perform respiratory flow-phase triggered closed-loop diaphragmatic stimulation with resultant changes in respiratory flow and tidal volume.
\end{abstract}

\section{CLOSED-LOOP SYSTEM FOR VENTILATORY CONTROL}

Approximately 270,000 Americans $(0.1 \%$ of the US population) have traumatic spinal cord injury (SCI), with 12,000 new injuries being reported each year [1]. The cervical cord is the most common site of injury (54\% of all cases), usually leading to partial or complete tetraplegia. Since respiratory muscles are innervated by phrenic motoneuron output from the C3 to C5 levels, people with high-level cervical SCI also have partial or complete loss of ventilatory control. Diseases of the respiratory system are the leading cause of death after SCI (66.9\% of these were cases of pneumonia) [2]. Most people with SCI that require ventilatory management are initially supported with positive pressuremechanical ventilation, which is associated with significant discomfort, diaphragmatic atrophy, atelectasis and barotrauma [3].

For over 40 years, electrical stimulation of the phrenic nerve for diaphragmatic pacing has been clinically accepted as an alternative for ventilator-dependent people with tetraplegia [4]. More recently, laparoscopic implantation of intramuscular electrodes to stimulate the diaphragm has been considered for patients without intact phrenic nerves or unilateral phrenic function [5]. Some of these systems are commercially available. For example, the current NeuRx diaphragmatic pacing system for human use has four intramuscular electrodes implanted in the diaphragm and one sub-cutaneous. The transdermal leads from the electrodes are

*Research supported by the National Institutes of Health (R01NS086088) and Agence National pour la Recherche (ANR-13-NEUC-0001) thorugh the US-French Collaborative Research in Computational Neuroscience program.

A.M. Zbrzeski, Y. Bornat, S. Renaud are with the Department of Bioelectronics in Laboratory IMS-CNRS UMR 5218, University of Bordeaux, e-mail: adeline.zbrzeski@ims-bordeaux.fr

R. Siu, B. Hillen and R. Jung are with the Department of Biomedical Engineering, Florida International University, Miami, FL 33134, email: rsiu001@fiu.edu, bhillen@fiu.edu, rjung@fiu.edu. connected to an external pulse generator. All commercially available systems employ an open-loop control system to deliver electrical impulses through the implanted electrodes $[6,7]$. Despite being widely used, open-loop control systems have the drawback that they require iterative manual tuning of stimulation parameters, and lack the ability to adapt to variable metabolic demands. A closed-loop adaptive control system could overcome these drawbacks.

Commercial closed-loop systems have been developed using mechanical ventilation [8]. However, they do not remain suitable for mobility or comfort and they could increase risks of tracheal problems. Enhancement of the openloop diaphragmatic systems currently used commercially could be a viable alternative. With the long-term goal of developing an implantable biohybrid closed-loop system, we are developing control algorithms and hardware that may ultimately be packaged into an implantable device. The viability of utilization of such hardware needs to be evaluated in a living system.

Previously diaphragmatic pacing studies have been conducted in large animal models (e.g. dog). However, the rodent model has been extensively used for studies on respiratory control. We have previously developed and utilized a rodent model for adaptive control of movement using functional electrical stimulation before and after incomplete spinal cord injury [9].

Here, we present the hardware prototype for development of a closed-loop control system for diaphragmatic pacing. The hardware platform has been developed for real-time recording and processing of biosignals [10]. Also, we present for the first time a rodent-model setup for flow-phase triggered closed-loop diaphragmatic stimulation.

\section{MAterials AND MethodS}

Fig. 1A illustrates the rodent-model setup for flow-phase triggered closed-loop diaphragmatic stimulation. Uninjured rats were used for the closed-loop validation preliminary study. Animal surgery and preparation are described in section II.A. A custom versatile system "Multimed" was developed for implementing the controller (section II.B, Fig. $1 \mathrm{~B} / \mathrm{C})$. The Multimed input sensor was connected to the amplifier output (Model 1700 Differential AC Amplifier, AM System). Also, Multimed controlled the parameters and trigger for activating a commercial stimulator (FNS-16, CWE Inc.).

Multi-stranded stainless steel wire (AS631, Cooner Wire Inc.) was used to fabricate the stimulation electrodes by modifying the design published in [11,12]. 


\section{A. Rodent model for Diaphragmatic Pacing}

Studies were performed on Sprague Dawley adult male rats $(n=2,6-7$ months old) weighing 520-535g. All experimental protocols were approved by the Florida International University Institutional Animal Care and Use Committee.

Rats were anesthetized with Pentobarbital ( $50 \mathrm{mg} / \mathrm{kg}$ ) and supplemental isoflurane. The animals were tracheotomized and connected to a breathing circuit containing a pneumotachometer (4120A, Hanns Rudolph). A $2 \mathrm{~cm}$ lateral incision was made into the left side of the abdominal cavity below the 12th rib. The electrodes were inserted distally into the costal region of the left hemi-diaphragm via a 22 gauge needle guide and connected to an electrical stimulator (FNS16, CWE Inc.). A pair of single stranded electrodes (Stainless Steel, AM Systems) placed subcutaneously over the pectoralis muscles were used to monitor the stimulation artifact.

The pneumotachometer was connected to a transducer amplifier (PM-1000, CWE Inc.) with an amplification sensitivity of $10 \mathrm{mV}$ and $10 \mathrm{~Hz}$-lowpass filter for obtaining the flow signal which was then integrated (PI-1000, CWE Inc., time constant of $0.2 \mathrm{~s}, 15 \mathrm{~Hz}$-low pass filter) to obtain the breath-by-breath volume. The flow and volume signals were sampled at $6 \mathrm{kHz}$ by a PC using a DAQ board (NI USB-6255, National Instruments) and saved using custom-developed software in LabView (2012 SPI, National Instruments).

\section{B. Multimed as flow-phase triggered closed-loop system}

Multimed is a versatile multi-application platform for real-time processing of biological signals and closed-loop systems. It has already been presented in a setup applied to in vitro recording of biosignals for diabetes applications [10, 13]. The Multimed hardware provides circuits for the acquisition of electrical biological signals using microelectrode arrays or classic electric inputs (SMA, BNC, etc.). The digital computation of the system is implemented on a Xilinx Spartan 6 FPGA (XC6SLX150).

It is associated with a library of processing modules, or 'IP's for Intellectual Property in design literature. These IPs perform in hardware highly parallel computation, and are dedicated to signal shaping (e.g. filters, integrators, derivators), event extraction from wave signals, event computation, stimulator control as outputs, real-time display and data storage. These IPs were designed to process data with a $10 \mathrm{kHz}$ sampling frequency, ensuring accurate biosignal reconstruction for preliminary investigations. For this experiment, the entire features of the system (computation, display and storage) only require $16 \%$ of the FPGA hardware resources. Fig. $1 \mathrm{~B}$ is a schematic of the implanted computational architecture for the early-stage version of the project.

The Multimed system is able to display any data of the computation architecture in real-time. The Multimed system has a dedicated display port to control a screen and avoid data transfer latency that can exceed 1s with a standard computer. The associated computer is then reduced to the minimal role of sending computation parameters.

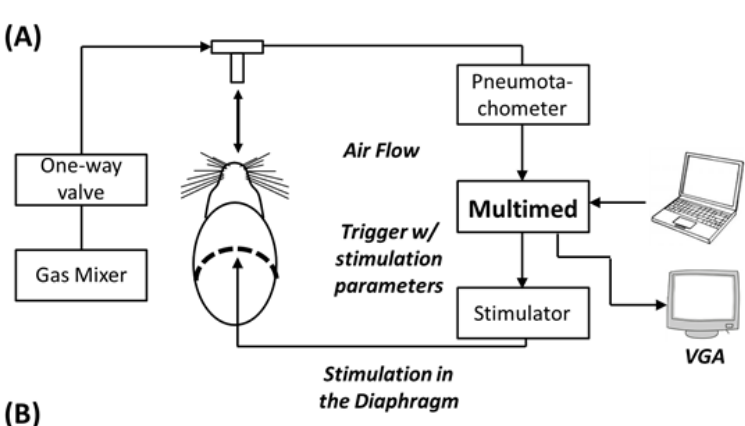

(B)

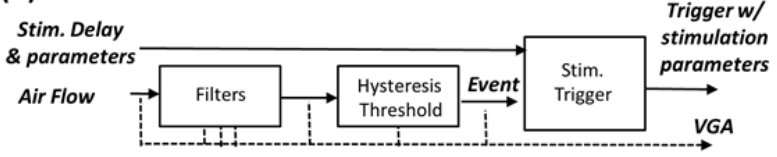

(C)

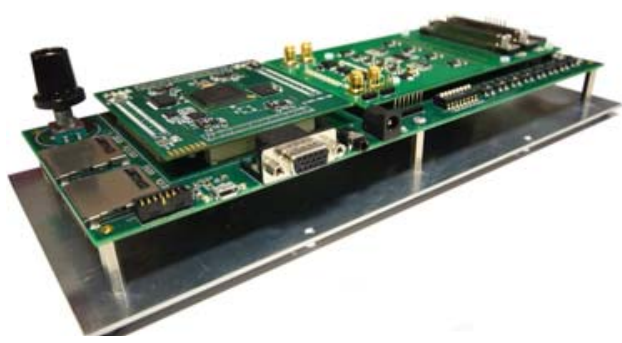

Figure 1. The rodent model setup for flow-phase triggered closed-loop diaphragmatic pacing. (A) Schematic of the experimental setup for evaluation of the flow-phase triggered closed-loop control system using the Multimed platform. The rodent can receive various gas mixtures to simulate various metabolic conditions. A $\mathrm{CO}_{2}$ analyser can be connected after the pneumotachometer if gas analysis is required (not shown here). Volume was extracted using an integrator after the pneumotachometer (not shown). The air flow was analysed by the Multimed to determin intiation of inspiration. Then, a delayed trigger was sent to the stimulator with appropriate stimulus parameters. (B) Schematic of the closed-loop system implemented on the Multimed platform. All signals and thresholds parameters can be displayed on a VGA (dashed lines) for real-time visual control. The system allows real-time update of all parameters: bandwidth and gain of the filters, hysteresis thresholds, the stimulation amplitudes, phase durations and frequency, and the trigger delay. (C) Photograph of the Multimed system.

Any threshold crossing is detected within $7 \mu$ s after sampling time and the longest delay duration is actually transferring eventual stimulation data and trigger to the stimulator through a RS232 link (3 to 4ms, depending on stimulation parameters). The system can switch to any other stimulation trigger protocol to overcome this limitation.

\section{Closed-loop Controller Settings}

On the Multimed, the air flow was filtered between 0.3 to $78 \mathrm{~Hz}$. Two types of settings were used for triggers. The first setting was used to create a trigger at a delay of 100 or $300 \mathrm{~ms}$ after the onset of inspiration. The second setting, referred to as "peak" trigger, was used to create a trigger at the maximum peak of the filtered flow with no delay. Thus, there were three flow-phase triggered stimulation paradigms.

After obtaining 30s of ventilator flow data, which represented at least 15 breaths, the stimulation was turned $\mathrm{ON}$ and diaphragmatic pacing was provided for each breath after the triggered event. Stimulation was done for $210 \mathrm{~ms}$ at $75 \mathrm{~Hz}$ using cathodic first, bi-phasic current stimulation pulses of $2.5 \mathrm{~mA}$ amplitude and pulse widths of $200 \mu \mathrm{s} /$ phase. 

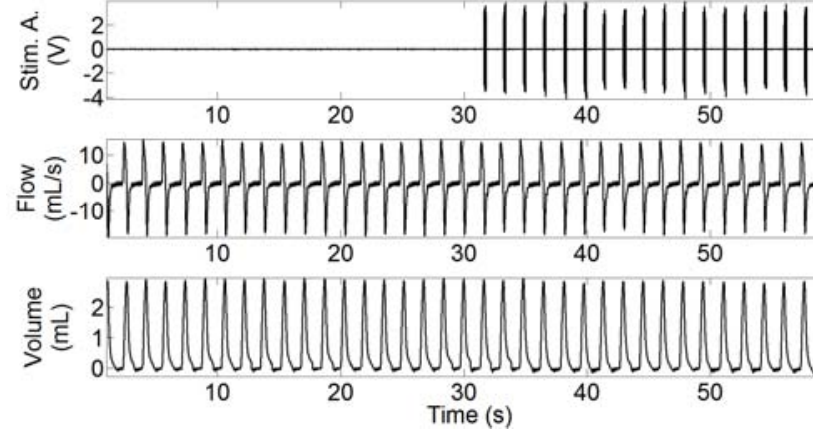

Figure 2. Sample of Flow and Volume recordings, with the 300ms-trigger stimulation. Stim.A is the is the recorded stimulation artifact.

TABLE I. BREATHING RATE \& VOLUME

\begin{tabular}{|c|c|c|c|}
\hline \multicolumn{2}{|c|}{ Controller Settings } & \multirow{2}{*}{$\begin{array}{c}\text { Breathing rate } \\
\text { (Br/min) }\end{array}$} & \multirow{2}{*}{$\begin{array}{l}\text { Volume per } \\
\text { Breath }(\mathrm{mL})\end{array}$} \\
\hline Stimulation & Trigger & & \\
\hline \multirow{3}{*}{ OFF } & "peak" & $37.61+/-0.54$ & $0.945+/-0.037$ \\
\hline & $100 \mathrm{~ms}$ & $37.06+/-0.45$ & $0.972+/-0.030$ \\
\hline & $300 \mathrm{~ms}$ & $37.12+/-0.62$ & $1.002+/-0.030$ \\
\hline \multirow{3}{*}{$\mathrm{ON}$} & "peak" & $38.37+/-0.71$ & $0.938+/-0.031$ \\
\hline & $100 \mathrm{~ms}$ & $37.11+/-0.61$ & $0.997+/-0.033$ \\
\hline & $300 \mathrm{~ms}$ & $36.96+/-0.69$ & $0.969+/-0.042$ \\
\hline
\end{tabular}
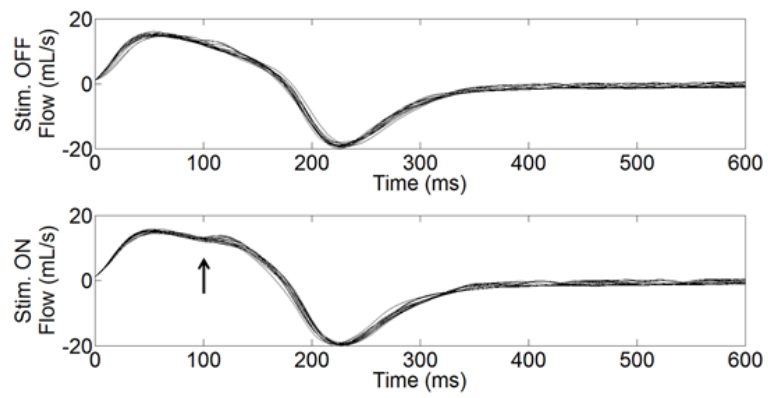

Figure 3. Example of 15 flow curves overlap (top) without and (bottom) with stimulation, with a 100ms-delay. The arrow marks the start of the stimulation.
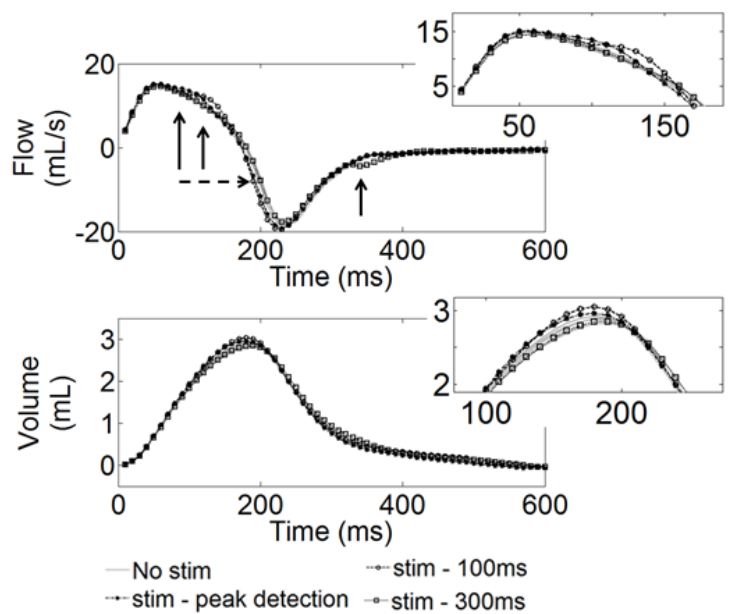

Figure 4. Averaged flow and volume without and with stimulation. Vertical plain arrows show stimulation starting time (Flow panel); horizontal dashed arrows illusrtrate shoertneing of the inpsiratory phase.
Stimulation amplitude was selected to visually alter the flow when the stimulation was sent at the event with no delay.

\section{Data Analysis}

To calibrate the volume, 1.0, 2.0 and $3.0 \mathrm{ml}$ syringes were connected to the flow head and the output from the integrator obtained for 35 to 45 strokes for each volume level. A 3point calibration was performed to confirm linearity. The relationship between the integrator output voltage and the volume for the air injected was determined and applied to all acquired respiratory volume data. The raw data sampled using the DAQ board was imported into Matlab ${ }^{\circledR}$ (The MathWorks, Natick, MA, USA) for data analysis and the breath-by-breath frequency and peak volume per breath (tidal volume) determined.

Input flow into the Multimed was digitally filtered using a $5^{\text {th }}$ order Butterworth low-pass filter at $50 \mathrm{~Hz}$. Volume was computed by Multimed by integrating the flow signal. Breath cycles were defined based on the inspiratory flow signals (positive deflection). An event was generated when the flow crossed a hysteresis threshold. This threshold was set above the remaining flow fluctuation on expiratory phases. Volume per breath was integrated using trapezoidal method. Mean, standard deviation and coefficient of variation were processed.

The stimulation artifact signal was recorded on the subcutaneous electrodes, and filtered with a $4^{\text {th }}$ order highpass filter at $750 \mathrm{~Hz}$. If the stimulation artifact signal was not null over a breath cycle, then the breath cycle was considered to have stimulation ON. For each stimulation paradigm 15 breath cycles were selected with stimulation OFF and ON.

\section{RESULTS}

Fig. 2 presents a 60 second sample of the filtered recorded breath-by-breath respiratory flow and volume data with and without the stimulation ON. Table 1 reports the average \pm standard deviation for the breathing rate and the integrated volume per breath for 15 breaths before (Stim OFF) and during stimulation (Stim ON) for one trial each for no delay from peak ("peak") and $100 \mathrm{~ms}$ and $300 \mathrm{~ms}$ delay from onset of inspiration.

Through visual monitoring of the flow and volume traces, an alteration of the waveform was observed when electrical stimulation of the diaphragm took place. As illustrated in Fig. 3, slight variation of the flow curve was observed when comparing overlapped curves for the 100ms-delay stimulations.

The flow and volume signals were also averaged on a breath-by-breath basis for 15 breaths before stimulation and 15 breaths after stimulation. Changes in flow and volume caused by stimulation were compared using these averaged waveforms. Fig. 4 illustrates the averaged flow and volume traces with or without stimulation for one trial for each stimulation paradigm. Variations in flow during the inspiratory and post-expiratory phases were identified (Fig.4, vertical arrows). Changes in flow were observed $\sim 30 \mathrm{~ms}$ after the stimulation was initiated during inspiration or $\sim 50 \mathrm{~ms}$ during expiration (vertical arrows in Fig. 4). Maximal increase in inspiratory flow to $2.27 \mathrm{~mL} / \mathrm{s}$ and $2.09 \mathrm{~mL} /$ 
occurred for stimuli applied at the "peak" or 100ms-delay stimulation events during inspiration while the expiratory flow changed to $2.06 \mathrm{~mL} / \mathrm{s}$ for the $300 \mathrm{~ms}$-delay stimulation event during expiration.

The average tidal volume increased from $2.925 \mathrm{~mL}$ to $3.046 \mathrm{~mL}$, and from $2.885 \mathrm{~mL}$ to $2.962 \mathrm{~mL}$, respectively for the $100 \mathrm{~ms}-$ delay and "peak" no-delay paradigms. The 300msdelay led to a slightly decreased tidal volume $(2.902 \mathrm{~mL}$ to 2.852mL).

\section{DISCUSSION}

This work presents an original closed-loop setup for diaphragmatic pacing using an uninjured rodent model and a versatile multi-application hardware data acquisition and processing platform for proof-of-concept demonstration.

In the rodent model used the current amplitude for stimulating the diaphragm could be reduced by optimizing the location of the stimulating electrode in relation to the neuromuscular junction. If all stimulation were to occur when the neural drive controlled the diaphragm's contraction, the proper placement of the electrode would provide synergistic stimulation and enhance muscle contraction without likely requiring high current values. Visual inspection of the implanted electrode post-mortem verified that the electrode had not dislodged from the diaphragm muscle.

The results of this original flow-phase triggered closedloop stimulation in a rodent model have shown that the system is ready to be evaluated using more complex adaptive controller paradigms. As presented in section 2, the current system takes less than $20 \%$ of resources. In addition, the longest delay (namely, the latency between the identification of a threshold and a trigger command) can be easily reduced from few milliseconds to few microseconds. Currently, it takes $30 \mathrm{msec}$ to $50 \mathrm{msec}$ for the commands to be relayed to the commercial FNS stimulator. The delay between an adaptive controller and the stimulator will be significantly improved by using our custom integrated circuit stimulator in development. Furthermore, the biosignal of this breathing application has slow dynamics, where a breath lasts for a minimum 650ms [14]. It is then possible to perform internal down-sampling, for example for a $1 \mathrm{kHz}$ sampling (1ms period). The processing time is inferior to $10 \mu \mathrm{s}$, so the current system would remain idle $99 \%$ of the time. This available time, associated with the remaining hardware resources of the FPGA, leaves room for much more complex real-time computation. Moreover, the system parameters can be easily adapted from rodent to human model with no impact on the processing performances, and the trigger could be based on other measures of ventilatory status. Therefore, the Multimed platform meets the needs for real-time closed-loop adaptive computation and especially for the experiments related to the control of the respiratory system.

\section{CONCLUSION}

We present an original experimental setup, where for the first time a rodent model for diaphragmatic pacing has been implemented. The preliminary data obtained using this rodent-model validate the functionality of the Multimed platform for assessing closed-loop control of diaphragmatic pacing.

The experimental data obtained will also be useful in the design and evaluation of adaptive control systems tested in computational models of ventilatory control after spinal cord injury in rodents or humans [15]. Finally, these preliminary experiments will serve as a test set for the validation of an adaptive controller for use with our custom stimulator that are, respectively, under development and electrical characterization.

\section{ACKNOWLEDGMENT}

We thank Mr. Ashwin Mangalore and Mr. Anil K. Thota for guidance with the experimental setup and Dr. James J. Abbas for discussion of concepts of adaptive closed-loop control using functional electrical stimulation.

\section{REFERENCES}

[1] NSCISC, "Spinal cord injury facts and figures at a glance," National Spinal Cord Injury Statistical Center, 2012

[2] NSCISC, "Annual Statistical Report - Complete Public Version," National Spinal Cord Injury Statistical Center, 2011

[3] A.F. DiMarco, "Restoration of respiratory muscle function following spinal cord injury: Review of electrical and magnetic stimulation techniques," Resp Physiol Neurobiol, vol. 147, pp. 273-287, 2005

[4] W.W. Glenn, M.L. Phelps, J.A. Elefteriades, B. Dentz, and J.F. Hogan, "Twenty years of experience in phrenic nerve stimulation to pace the diaphragm,” Pacing Clin Electrophysiol, vol. 9, pp. 780-4, Nov 1986

[5] R.P. Onders, M. Elmo, S. Khansarinia, B. Bowman, J. Yee, J. Road, B. Bass, B. Dunkin, P.E. Ingvarsson, and M. Oddsdottir, "Complete worldwide operative experience in laparoscopic diaphragm pacing: results and differences in spinal cord injured patients and amyotrophic lateral sclerosis patients," Surg Endosc, vol. 23, pp. 1433-40, Jul 2009

[6] A.F. DiMarco, "Phrenic nerve stimulation in patients with spinal cord injury," Resp Physiol Neurobiol, vol. 169, pp. 200-9, Nov 302009

[7] A. Madsen (2012, 3 Jan.). "A Closer Look: Diaphragm Pacing System”. MDA/ALS Newsmagazine, vol.17, No.1 [Online]. Available: http://alsn.mda.org/article/closer-look-diaphragm-pacing-system [Accessed: Nov. 10, 2014].

[8] R.L. Chatburn and E. Mireles-Cabodevila, "Closed-loop control of mechanical ventilation: Description and classification of targeting schemes,” Respiratory Care, vol. 65, pp. 85-102, Jan 2011

[9] M. Fairchild, S.J. Kim, A. Iarkov, J.J. Abbas, R. Jung. "Repetitive hindlimb movement using intermittent adaptive neuromuscular electrical stimulation in an incomplete spinal injury rodent model,” Exp Neurology, vol. 223, pp. 623-633, June 2010.

[10] A. Quotb, J.-B. Floderer, T. Bollengier, R. Fabre, S. Renaud, and Y. Bornat, "Live Demonstration: Real-time wavelet spike detection with in vitro biological signals," IEEE BioCAS, p. 79, Nov 2012

[11] R.B. Trelease, G.C. Sieck, R.M. Harper, "A new technique for acute and chronic recording of crural diaphragm EMG in cats," Electroencephalogr Clin Neurophysiol, vol. 53, pp. 459-62, Apr 1982

[12] D.E. Dow, C.B. Mantilla, W.Z. Zhan, G.C. Sieck, "EMG-based detection of inspiration in the rat diaphragm muscle," IEEE Eng Med Biol Soc, vol. 1, pp. 1204-7, 2006

[13] A. Quotb, Y. Bornat, M. Raoux, J. Lang, and S. Renaud, "NeuroBetaMed: A re-configurable wavelet-based event detection circuit for in vitro biological signals," IEEE ISCAS, pp.1532-35, 2012

[14] C.B. Mantilla, Y.B. Seven, W.-Z. Zhan, and G. C. Sieck, "Diaphragm motor unit recruitment in rats,” Resp Physiol Neurobiol, vol.173, pp.101-106, 2010

[15] B.K. Hillen, R. Jung, "Computational model of human ventilation for electrical stimulation following cervical spinal cord injury,” $B M C$ Neuroscience 2014, 15(Suppl 1):P133 (21 July 2014). 